\title{
Modeling and Control of Advanced Propulsion Systems
}

\author{
L. Guzzella \\ ETH Zurich, 8092 Zurich, Switzerland \\ email: Iguzzella@ethz.ch
}

\begin{abstract}
Resumé - Modélisation et contrôle des systèmes de propulsion avancés - Les systèmes de propulsion pour les véhicules routiers d'aujourd'hui comportent différents sous-systèmes responsables de la conversion et du stockage de l'énergie. Ces systèmes complexes ne peuvent pas être optimisés pour la performance, pour l'économie de carburant, et pour le coût en adoptant une approche "essai-erreur". Il faut employer des méthodes systématiques d'optimisation à base de modèles mathématiques qui décrivent le comportement du système étudié avec un niveau de précision approprié. Cet article récapitule des idées générales parmi les plus efficaces pour résoudre ce genre de problèmes et illustre les méthodes présentées par trois exemples d'application.
\end{abstract}

\begin{abstract}
Modeling and Control of Advanced Propulsion Systems - Propulsion systems of modern road vehicles include several subsystems for energy conversion and storage. Such complex systems cannot be optimized for performance, fuel economy, and cost using "trial-and-error" approaches. Systematic optimizations must be performed using mathematical models that approximate the system's behavior on the appropriate precision level. This survey paper describes some of the most useful general ideas in this field and shows three examples of their application.
\end{abstract}

\section{INTRODUCTION}

The number of road vehicles (in particular automobiles) is inexorably increasing. While several regions experience market saturations, many countries are faced with a dramatic increase of the number of passenger cars. This trend creates an enormous demand for (fossil) fuels, whose price increases accordingly. Moreover, serious concerns regarding the influence of $\mathrm{CO}_{2}$ emission on global climate change have been raised by many. It is therefore of paramount importance to develop new propulsion systems that produce the necessary mechanical work with a much improved fuel economy. Pollutant emissions from internal combustion engines (ICE) have been reduced by orders of magnitude in the last decades. Therefore, these aspects will be of less importance in the future and, although several problems await a solution in this area as well, this article focuses on fuel economy.

\section{ANALYSIS OF STANDARD SYSTEMS}

Modern power train subsystems both standard (gasoline or Diesel engines, manual or automatic gear boxes, etc.) and nonstandard (electric traction motors, batteries, etc.) have reached a remarkable maturity and it will be rather difficult to improve on their peak efficiencies. The main open problems, whose solutions would entail a substantial improvement in fuel economy, are on the systems level. 
In particular:

1 At idle or in deceleration (coasting) phases the propulsion system is not shut down and decoupled from the vehicle and, therefore, induces large friction losses.

2 Compared to their peak efficiency the partload efficiency of standard propulsion systems is substantially lower (factor two and more).

3 Standard brakes dissipate the vehicle's kinetic energy irreversibly to heat, which is then lost to the environment.

4 Each vehicle remains isolated; no information about its actual and future driving profile and the traffic situation in which it operates is used to optimize the fuel economy. In particular the several energy reservoirs present in the vehicle can be used for that purpose. Even standard automobiles have at least two energy reservoirs: the fuel tank and the vehicle mass that "stores" kinetic energy. Advanced power trains include more reservoirs, such as batteries, super capacitors, etc.

These drawbacks can be reduced substantially by a welldesigned control system. Optimal feed forward control action is the main tool needed to achieve the objectives in fuel economy. Of course appropriate actuators and, in the case when feedback action is employed as well, suitable sensors must be available in the power train system.

The process to synthesize such optimal control systems is not trivial. Due to the substantial system complexity a purely experimental approach will, most likely, fail. The only option that holds some promise of success is a model-based and computer-assisted optimization. Appropriate models and optimization algorithms (gradient-based or others) are the only viable approach.

\section{MODELING PARADIGMS}

Complex system structure and long optimization horizons induce high computational costs such that models that can be used for the purpose of optimizing the fuel economy of propulsion systems must be rather simple. The number of "states" (energy and other reservoirs) must be very low and a moderate number of discretization intervals, if needed, must suffice to obtain precise results.

Typically, models are formulated using sets of ordinary differential equations such as:

$$
m \frac{d}{d t} v(t)=-\left[k_{0}+k_{1} v^{2}(t)\right]+F(t)
$$

that approximate the longitudinal dynamics of a road vehicle as illustrated in Figure 1. Such equations are useful for the synthesis of feedback control systems, the formulation of optimal control problems, etc. Such "forward" descriptions, which correctly include the physical causalities, induce a relatively large computational burden.

An alternative approach is to use a "backwards" formulation, which, of course, is only applicable when the trajectory $v(t)$ is known a priori.

$$
F\left(t_{i}\right) \approx m \frac{v\left(t_{i}\right)-v\left(t_{i-1}\right)}{\delta}+k_{0}+k_{1}\left(\frac{v\left(t_{i}\right)+v\left(t_{i-1}\right)}{2}\right)^{2}
$$

Such a description induces substantially lower computational cost and is well suited to problems with long time horizons.

\section{OPTIMIZATION METHODS}

Traditionally, the following three optimization approaches have been used in technical applications:

- Nonlinear programming:

A gradient-based search in a finite-dimensional parameters space.

- Dynamic programming:

A discretization of the time and the variable space of an optimization problem, and its solution using "Bellman's optimality principle.". This principle states that on an optimal trajectory each intermediate point may be defined as a new initial condition. In this case the rest of the original optimal trajectory is the optimal solution for this new initial point. This simple observation permits to solve the problem "backwards". Of course, this is only possible if the complete driving profile is known a priori.

\section{- Optimal control:}

Similar to calculus of variation, but with the additional possibility to include constraints on the input signal ("Pontrjagin's minimum principle").

Instead of giving abstract descriptions of these methods, which can be found in [1], three examples are shown below that illustrate the application of these methods for the design of power train systems.

\section{SOME EXAMPLES}

In this section three examples are presented briefly. The first, a simple parameter optimization problem, illustrates the optimal choice of the duty cycling parameters of a parallel hybrid

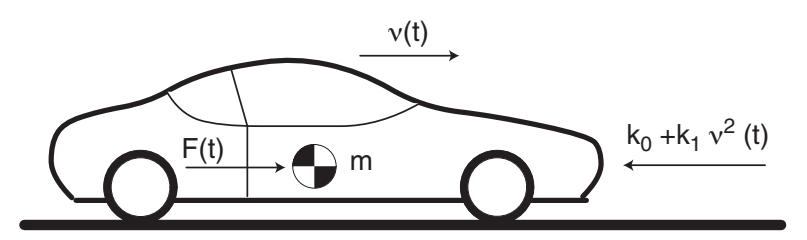

Figure 1

Main parameters and variables needed to describe the longitudinal dynamics of a road vehicle. 


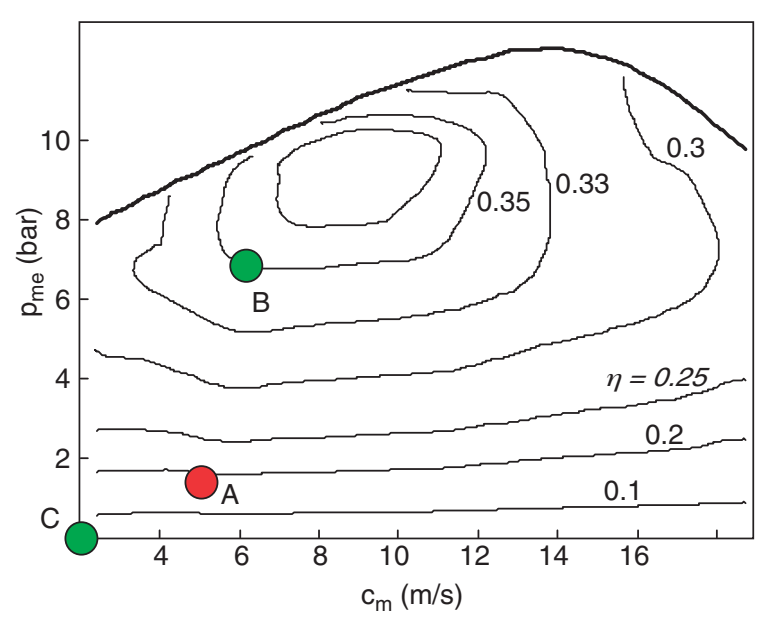

Figure 2

Map of a standard SI engine; $c_{m}$ mean piston speed (normalized engine speed), $p_{m e}$ mean effective pressure (normalized engine torque).

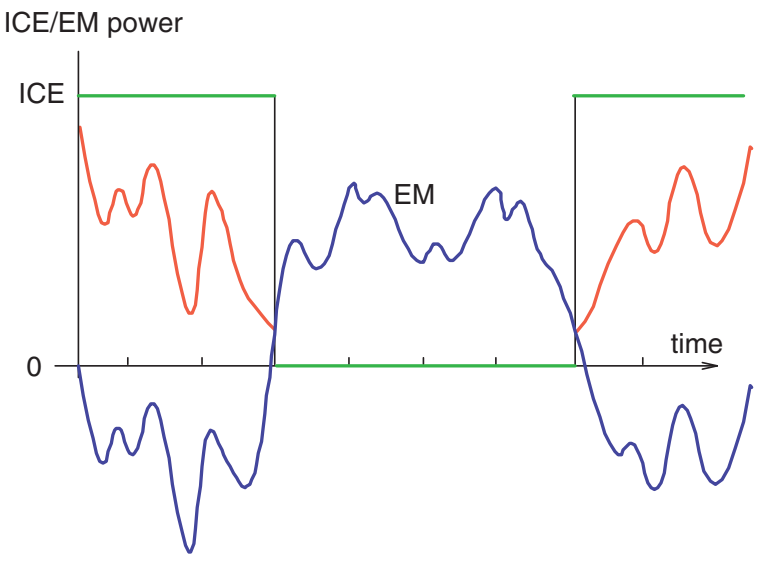

Figure 3

Duty cycle power distribution. ICE = internal combustion engine, $\mathrm{EM}=$ electric motor. vehicle. The second example shows how the driving strategy of a HEV can be optimized when the future driving profile is known a priori using a dynamic programming approach. The third example shows an optimal control approach for the solution of a similar problem.

\subsection{Example: Optimal Duty Cycle}

This example shows the simplest approach to optimization of power train control systems. Figure 2 illustrates the main problem of most modern engines, i.e., the sharp decrease of efficiency when moving away from the high-load operating conditions. In regular driving conditions (point " $\mathrm{A}$ " in the engine map shown in Fig. 2) the efficiency can be substantially lower than in the best point (by a factor of two and more).

A much better approach is to "duty cycle" the engine, i.e., to operate it only in the point " $\mathrm{B}$ " or " $\mathrm{C}$ " in the map shown in Figure 2. In the first operating point the mechanical power is produced in the (almost) best possible conditions, in the second the engine is shut off and the vehicle is driven by the electric motor that is fed by an electric short-term storage device (either supercaps or batteries). The objective is to derive an optimal duty cycle timing, which realizes the best compromise between gains in engine efficiency and additional losses in energy conversion process (fuel $\rightarrow$ mechanical $\rightarrow$ electric $\rightarrow$ electrochemical $\rightarrow$ electric $\rightarrow$ mechanical).

To be more explicit, assume that the fuel energy consumed over a given cycle can be modeled as follows (for the sake of simplicity, the engine and the motor speed are assumed to be the same below):

$$
E_{f}^{c}=\int_{c y c l e} \frac{\omega \cdot T_{e}(u(x))}{\eta_{e}(\omega, u(x))} d x+\int_{c y c l e} \frac{\omega \cdot T_{m}(u(1-x))}{\eta_{m}(\omega, 1-u(x))} d x
$$

where $T_{\ldots}$ is the torque, and $\eta_{\ldots}$ the efficiency of the engine $(\ldots=e)$ and the electric motor $(\ldots=m)$. The control variable $u$ can be parametrized as piecewise constant in the interval $x \in\left[x_{i}, x_{i+1}\right)$ of the cycle and the unknown values $u_{i}$ are to be found by numerical optimization ("nonlinear programming"). The result will then lead to a system behavior similar to the one shown in Figure 3.

Of course such an optimization approach is feasible only for relatively simple "cycles" (piecewise constant vehicle speeds, etc.), because nonlinear programming algorithms are restricted to relatively few degrees of freedom (see [2]). If long cycles are to be analyzed, the methods presented in the next section are much better suited.

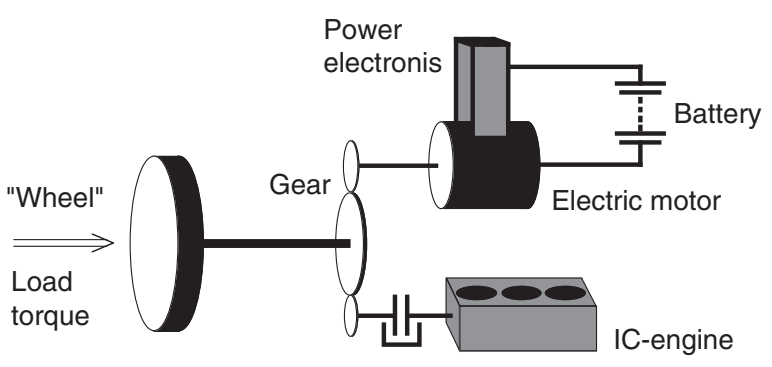

Actuated clutch

Figure 4

Layout of the ESORO Twin-Track power train structure. 


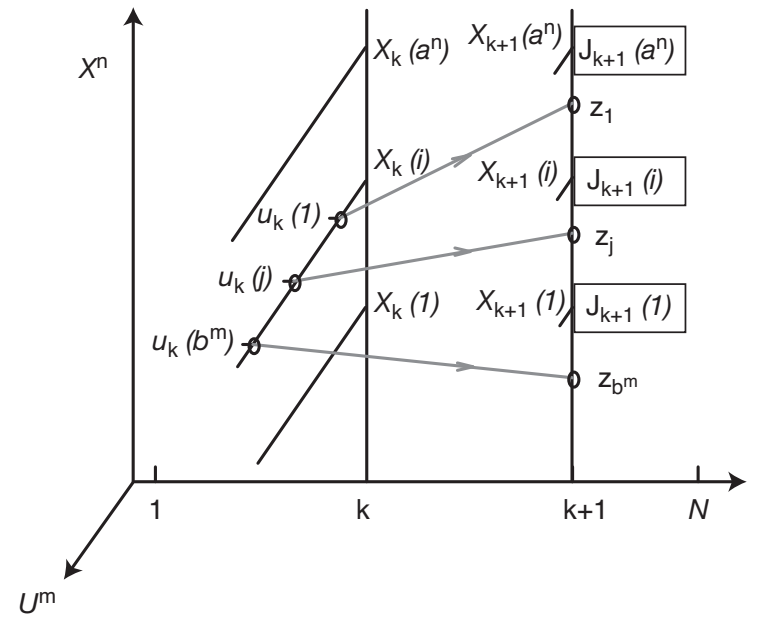

Figure 5

Main iteration step in a DP optimization.

\subsection{Example: Dynamic Programming}

The power train analyzed in this example is shown in Figure 4 This very simple parallel hybrid power train was actually built with the in intention to obtain first experiences with advanced control concepts. A detailed description of these ideas can be found in [3].
Since this power train is so simple one can test well totalcycle ("non-causal") optimization strategies. In this specific case a dynamic programming (DP) approach was chosen.

Figure 5 shows the key iteration step. The numerical "cost" of such computations increases with:

$$
O \approx N \cdot a^{n} \cdot b^{m}
$$

where $N$ is the number of time steps, $n$ the number of "states" (energy reservoirs), $m$ the number of control signals, and $a$ and $b$ the number of quantization points in the states and in the control signals, respectively. The important fact is that these cost increase only linearly with the time horizon, such that even "long" cycles can be handled with this approach. Figure 6 shows such a "long cycle" including speed and elevation information.

For such cycles the optimal control signals can be computed and compared to typical heuristic algorithms ("electric assist" is a popular one, see [4]). Figure 7 shows the result of such an analysis. The gains in fuel economy obtained going through the cycle are significant, but not very large. However, if the vehicle drives back along the same cycle and if the battery can be recharged from the electric grid ("plugin hybrid") then very large improvements in fuel economy can be achieved by managing the several energy reservoirs (fuel tank, battery, and kinetic and potential energy of the vehicle).
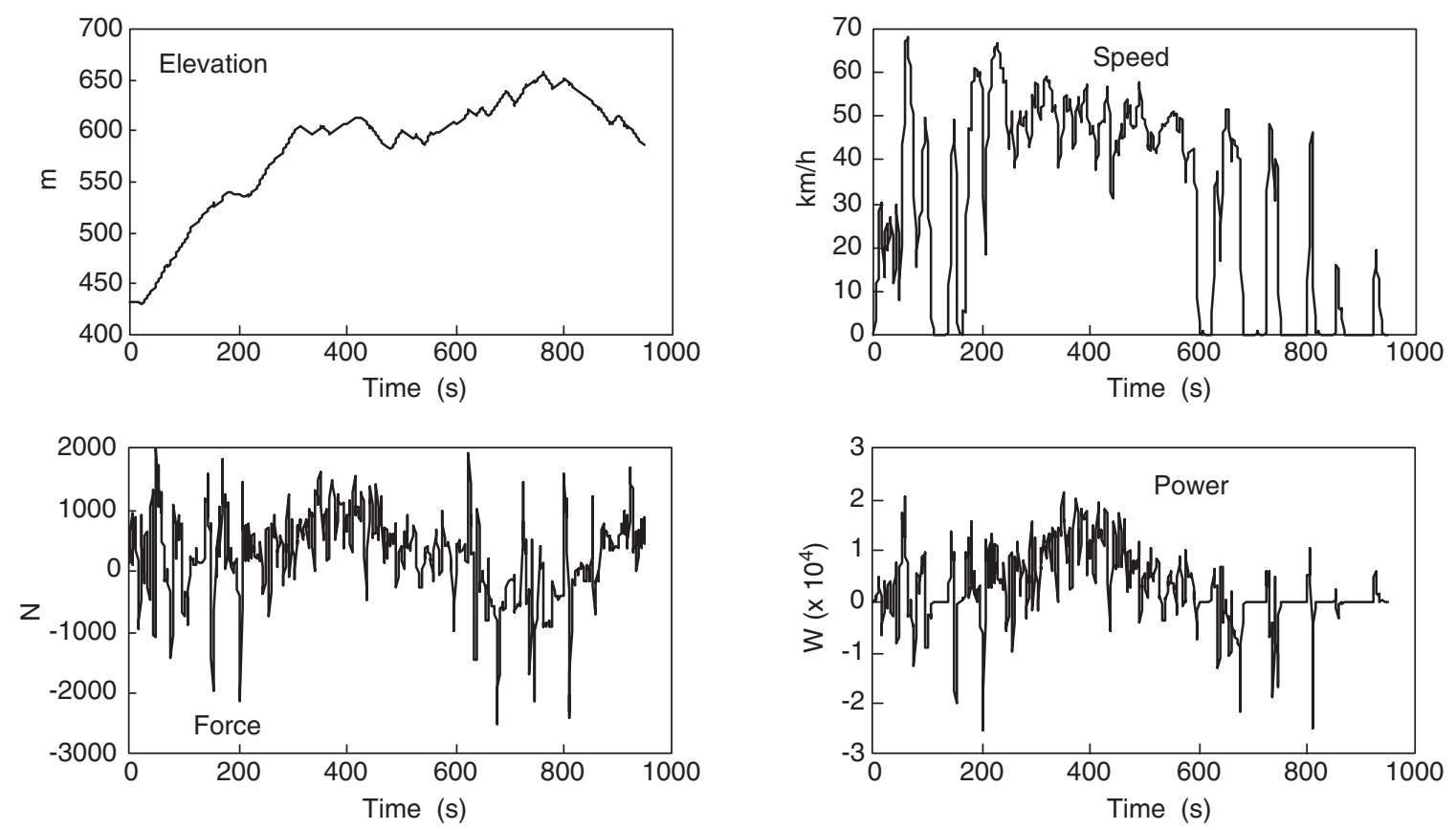

Figure 6

Elevation, speed, traction force and traction power of a real driving cycle. 

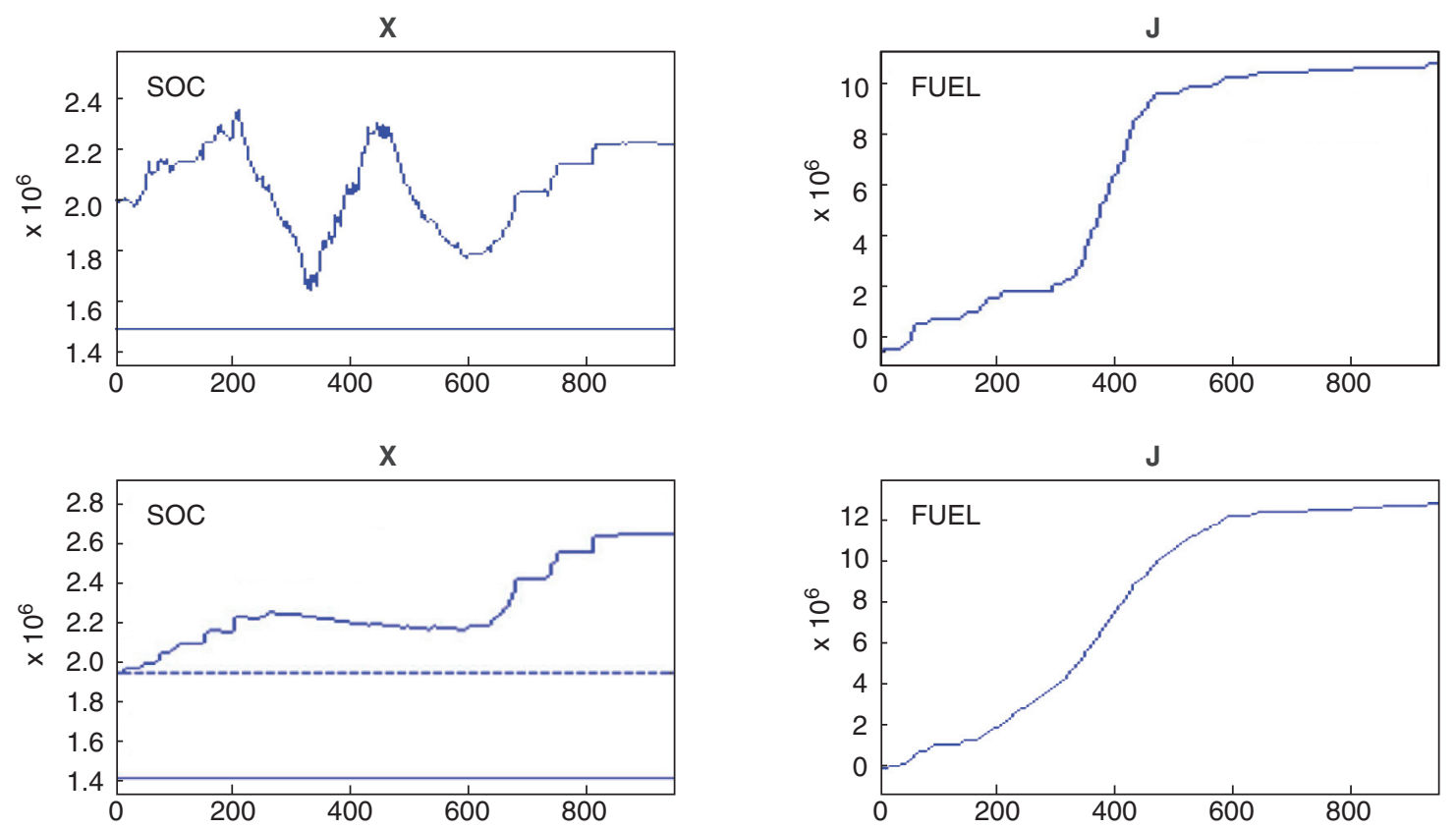

Figure 7

Top plot: optimal solution (SOC $=$ state of charge of the battery), bottom plot: heuristic solution .

\subsection{Example: Optimal Control}

The vehicle analyzed in this example study, called "PAC Car II," was designed for the Shell Eco-marathon events, which are competitions that reward the lowest fuel consumption. An extended version of this section was first published in [5]. The PAC Car II vehicle is equipped with a fuel cell electric power train. To compare its hydrogen consumption to cars that use liquid hydrocarbons, the lower heating value of those fuels is taken as the reference.

Besides the optimization of each component of the vehicle and its power train, the driving strategy had to be carefully chosen in order to achieve the best possible fuel economy. This optimization is the topic of the analysis presented below. First a short description of the main features of the vehicle is given. Then, starting from the formulation of the optimal control problem (OCP) various aspects of the optimization of the driving strategy are discussed in this case study.

With such an optimized driving strategy PAC Car II won the Nogaro (F) Shell Eco-marathon race in May 2005. A fuel consumption of $7 \mathrm{~g}$ of hydrogen per $100 \mathrm{~km}$ was recorded in that race (using the lower heating values of hydrogen and gasoline, this corresponds to an equivalent mileage of 3836 $\mathrm{km}$ per liter gasoline).

In the Shell Eco-marathon in Ladoux (F), one month later the same vehicle set a new world record for fuel-efficient ground vehicles by achieving an equivalent mileage of $5385 \mathrm{~km}$ per liter gasoline ( $5 \mathrm{~g}$ of hydrogen per $100 \mathrm{~km}$ ).

\subsubsection{Description of PAC Car II}

The PAC Car I and II vehicles were developed in the years 2000 to 2005 by a team of mechanical engineering graduate and undergraduate students at ETH Zurich. Figure 8 shows a picture of PAC Car II taken during the Nogaro 2005 event.

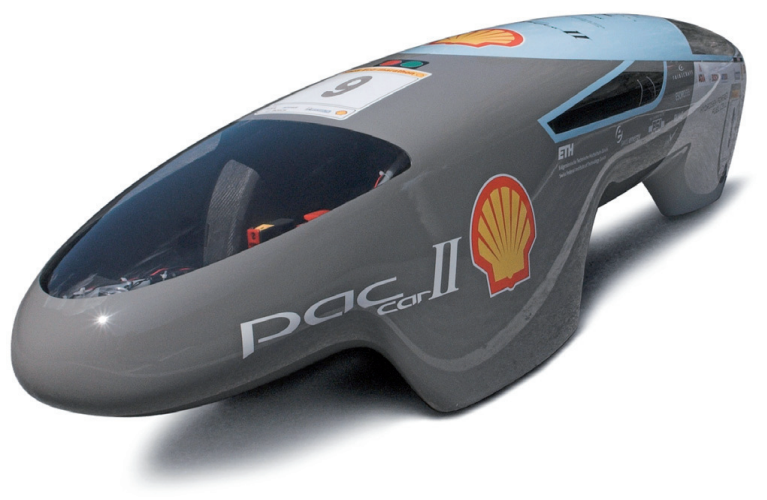

Figure 8

The PAC Car II vehicle. 
The vehicle was designed and manufactured according to the rules defined by the Shell Eco-marathon organizers. The main parameters are listed in Table 1. Two optimized directcurrent motors drive the vehicle. The electric energy is produced by a fuel cell system fed by compressed hydrogen. The high-level control of the power train, i.e., the control of the fuel cell system, the monitoring of important data in the vehicle, and the realization of the driving strategy are performed by an onboard computer system. High-efficiency $\mathrm{DC} / \mathrm{DC}$ converters that distribute the electric power to the high-performance electric motors are connected to the control system via an optical link.

TABLE 1

Main Parameters of the PAC Car II Vehicle

\begin{tabular}{l|c}
\hline Front area: & $A_{f}=0.254 \mathrm{~m}^{2}$ \\
\hline Aerodynamic coefficient: & $c_{d}=0.075$ \\
\hline Mass vehicle: & $m=29 \mathrm{~kg}$ \\
\hline Rolling friction coefficient: & $c_{r}=0.001$ \\
\hline
\end{tabular}

The total force $F_{\text {tot }}$ that acts on a road vehicle is the sum of the propulsion force $u$, the aerodynamic resistance $F_{a}$, the rolling resistance $F_{r}$, and the gravitational force $F_{g}$ :

$$
\begin{aligned}
F_{\text {tot }} & =u-F_{a}-F_{r}-F_{g} \\
& =u-\frac{\rho}{2} A_{f} c_{d} v^{2}-\left(c_{r} \cos (\gamma)+\sin (\gamma)\right) m g
\end{aligned}
$$

where the variable $\gamma$ stands for the inclination of the track at the actual position $x$ ( $g$ stands for the earth gravitational acceleration). More detailed models are known [4], but such descriptions are not needed for the purposes discussed in the following sections.

\subsubsection{Optimal Control Problem}

The problem of driving on a specific track while minimizing the fuel consumption can be formulated as an optimal control problem [1]. In a first step, the force at the wheel is minimized. Using Newton's law and Equation (1), the following equations define the dynamics of the OCP:

$$
\begin{aligned}
& \dot{x}=v \\
& \dot{v}=c_{1} u-c_{2} v^{2}-c_{3}-c_{3} \gamma
\end{aligned}
$$

As mentioned above, the traveled distance is symbolized by $x$, the vehicle speed by $v$, and the slope of the track by $\gamma(x)$. The coefficients $c_{i}$ immediately follow from Equation (1) dividing all constant terms by the vehicle mass $m$.

Braking the vehicle always results in a loss of energy and thus must be avoided under all circumstances. Accordingly, the force at the wheels may be only between zero and a maximum value $u \in\left[0, u_{\max }\right]$. The objective function, which has to be minimized, is the total work at the wheel over the demanded time $t_{b}$ :

$$
J(u)=\int_{0}^{t_{b}} u(t) v(t) d t
$$

The Hamiltonian associated to this OCP can be written as:

$$
H=u v+\lambda_{1} v+\lambda_{2}\left(c_{1} u-c_{2} v^{2}-c_{3}-c_{3} \gamma(x)\right)
$$

Since the problem is time-invariant, the Hamiltonian has to be constant. The differential equations that define the dynamics of the costate vector are:

$$
\begin{aligned}
& \dot{\lambda}_{1}=\lambda_{2} \cdot c_{4} \cdot \frac{\partial \gamma(x)}{\partial x} \\
& \dot{\lambda}_{2}=-u \cdot \lambda_{1}+2 \cdot \lambda_{2} \cdot c_{2} \cdot v
\end{aligned}
$$

According to the minimum principle, the Hamiltonian has a global minimum for the optimal control input:

$$
H\left(x_{\text {opt }}, v_{\text {opt }}, \lambda_{1 \text { lopt }}, \lambda_{2 o p t}, u_{\text {opt }}\right) \leq H\left(x_{\text {opt }}, v_{\text {opt }}, \lambda_{1 \text { opt }}, \lambda_{2 o p t}, u\right)
$$

Taking into account the constraints for the input, the following control strategy can be defined:

$$
u_{o p t}=\left\{\begin{array}{cc}
0 & \text { if } v+\lambda_{2} \cdot c_{1}>0 \\
u_{\sin g} & \text { if } v+\lambda_{2} \cdot c_{1} \equiv 0 \\
u_{\max } & \text { if } v+\lambda_{2} \cdot c_{1}>0
\end{array}\right.
$$

Below, the variable $h=v+\gamma_{1} \cdot c_{1}$ will be referred to as the switching function. When $h \equiv 0$ for a finite interval of time, the optimal control input cannot be derived directly from the switching function and the system is on a singular arc. In this case the derivatives of $h(t)$ with respect to time have to be used to find the input $u(t)$.

The first derivative of $h(t)$ doesn't yield the desired result, but the second derivative of $h(t)$ explicitly depends on $u(t)$. With this the singular arc follows as:

$$
u_{\text {sing }}=\frac{1}{c_{1}} \cdot\left(c_{2} \cdot v^{2}+c_{3}+c_{4} \cdot \gamma \cdot(x)\right)
$$

Using the model Equation (1) it is straightforward to show that this control produces a constant vehicle velocity $v(t)$.

\subsubsection{Optimization of the Driving Strategy}

The solution of the OCP showed that only three types of control signals need to be considered, viz. full load (boost), keeping the speed constant (sustain) and no power (coast). Using these three possibilities, some sub-problems are now to be discussed in order to find a solution to the full problem.

\section{Case: Small Elevations, Full Cycle}

Since the aerodynamic resistance is quadratic in the velocity, it is obvious that as long as the downhill forces do not exceed the sum of rolling and aerodynamic frictions, the smallest constant speed which exactly satisfies the averagespeed boundary condition will be the solution for the infinitehorizon OCP. For a finite horizon it might be preferable to 


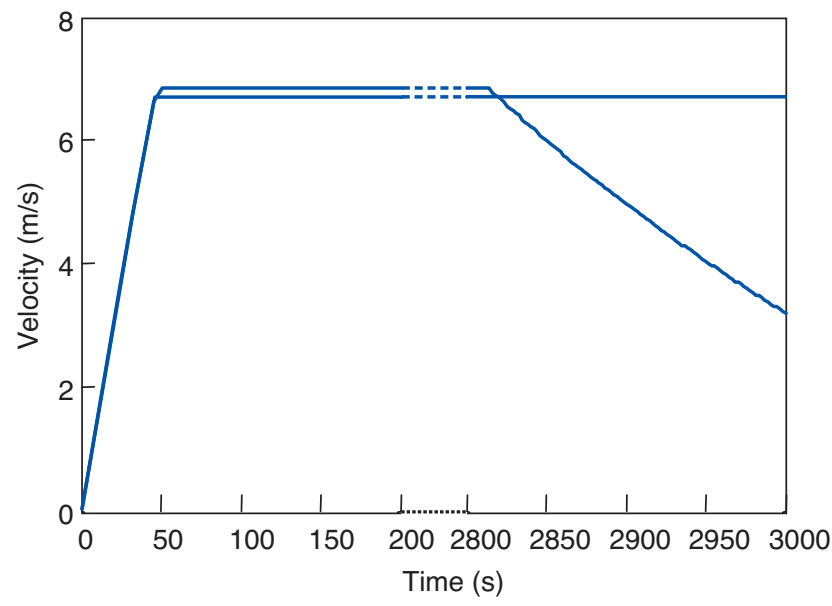

Figure 9

Speed traces for the two different strategies to solve the "small elevations" problem.

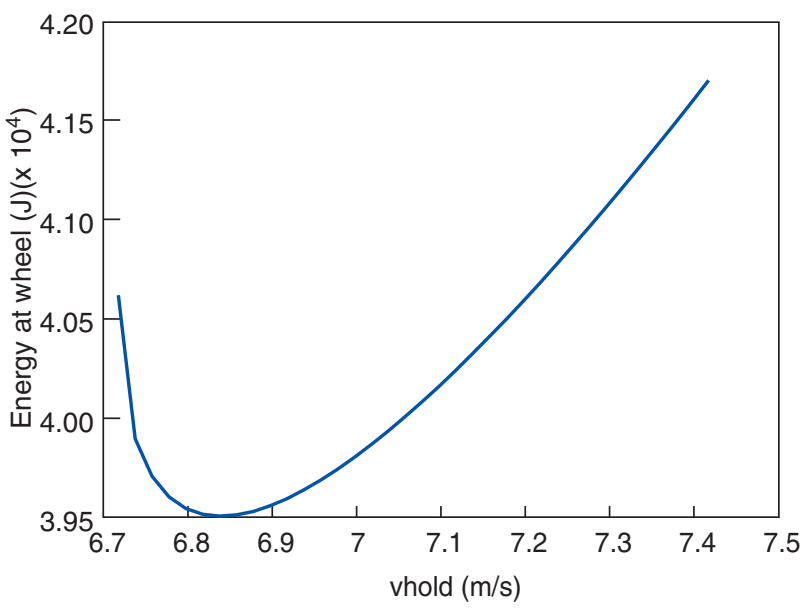

Figure 10

Integrated energy at the wheel for varying sustained speeds. sustain a slightly higher speed and to recover some of the kinetic energy by adding a coasting phase at the end. This problem is analyzed first because it can be solved without being forced to solve a two-point boundary-value problem; a simple parameter optimization suffices to find the optimal driving strategy.

Figure 9 shows the traces of the velocities for the infinite-horizon strategy and the optimized finite-horizon strategy for a specific average-speed situation. Figure 10 illustrates the demanded energy at the wheel for varying sustained speeds. The smallest sustained speed corresponds to the infinite-horizon strategy. Thus, in this specific case, reducing the end speed to approximately half of the sustained speed results in a $2.8 \%$ reduction of the demanded energy.

\section{Case: Large Elevations, Infinite Horizon}

For an aerodynamically optimized vehicle even small elevations can result in acceleration when driving downhill. Thus the force at the wheels can be set to zero and the control strategy has to be changed.

Here, the infinite-horizon problem for a specific racing track is discussed. The racing track chosen is the motor speedway in Rockingham (UK). Its elevation profile is illustrated in Figure 11. An average minimum speed of $v_{\text {avg,des }}=6.7 \mathrm{~m} / \mathrm{s}$ is required by the race regulations. The length of one lap on the track is $s_{\text {lap }}=2244 \mathrm{~m}$.

Knowing the only possible form of the optimal control signals, the following driving strategy is proposed:

- starting with an initial velocity $v_{0}$, a coasting phase is chosen first since the slope of the trace at the start line is known to be negative,
- at the time $t_{1}$ the control is switched on to keep a constant speed $v_{\text {sust }}$, and

- at the time $t_{2}$ the motors are shut off again until $t_{\text {end }}$ is reached.

The unknown variables $v_{0}, t_{1}, t_{2}$, and $t_{\text {end }}$ are chosen such that the energy at the wheel is minimized and that the boundary conditions:

$$
\begin{gathered}
v\left(t_{\text {end }}\right)=v_{0}, \\
s\left(t_{\text {end }}\right)=s_{\text {lap }}, \\
v_{\text {avg.dem }}=s\left(t_{\text {end }}\right) / t_{\text {end }}
\end{gathered}
$$

are satisfied.

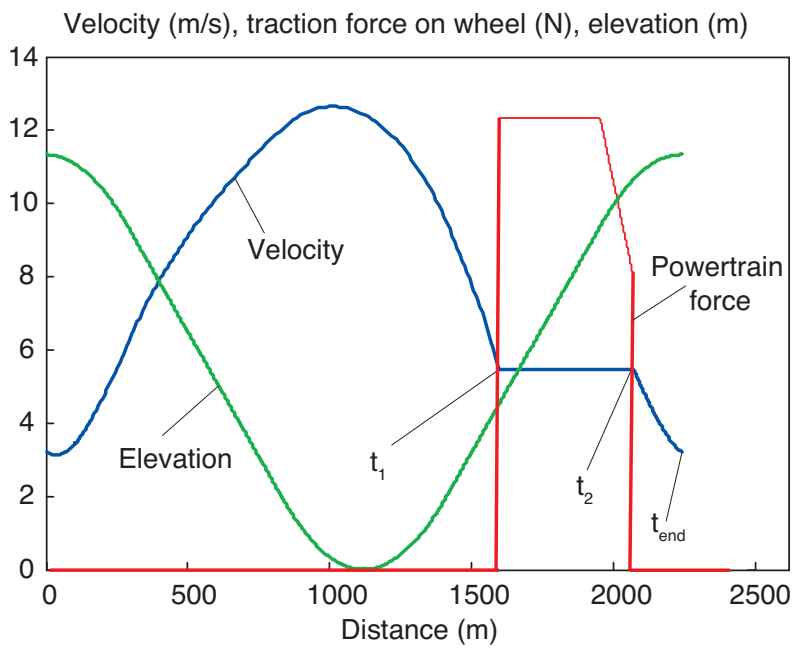

Figure 11

Elevation profile, optimal vehicle speed, and force at the wheel calculated for the Rockingham racing track. 


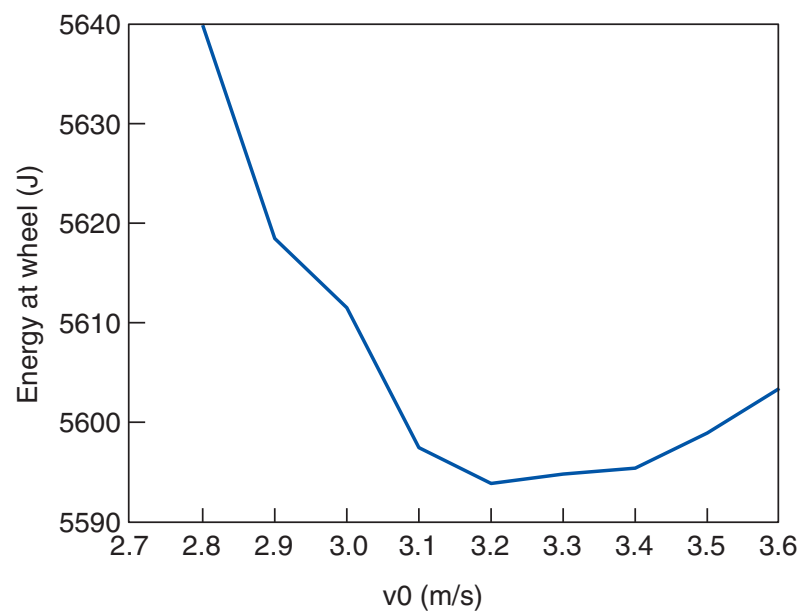

Figure 12

Mechanical energy consumption for varying initial speeds.

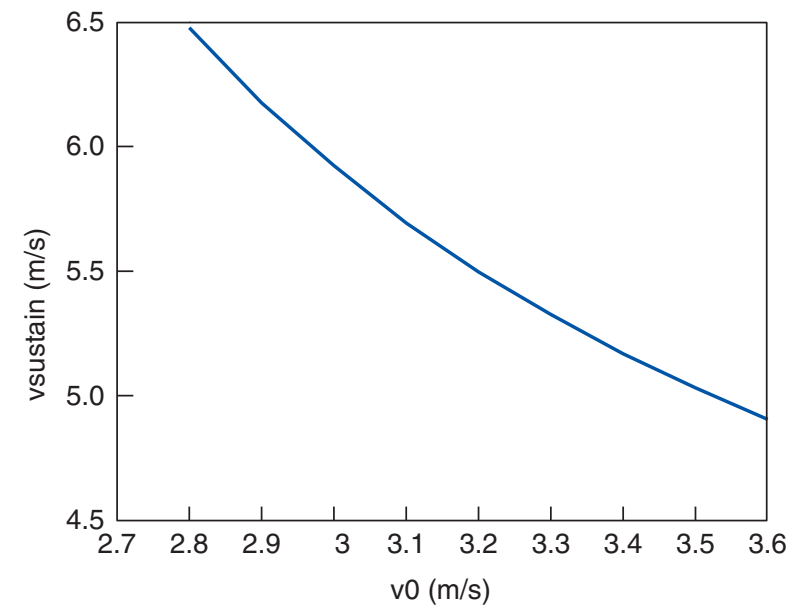

Figure 13

Optimal sustained speeds for varying initial speeds.
The solution of this numerical optimization problem with four unknown variables and three constraints poses quite some problems to standard numerical solvers. Therefore another method is proposed:

- choose $v_{0}$ as the independent variable of the optimization problem,

- use $v_{\text {sust }}$ to satisfy the boundary conditions,

- integrate the differential equations forward starting with $v(0)=v_{0}$ until $v_{\text {sust }}$ is reached with decreasing velocity; this yields the two unknown variables $t_{1}$ and $s\left(t_{1}\right)$,

- integrate the system equations backwards starting with $t=s_{\text {lap }} / v_{\text {avg.dem }}, s=s_{\text {lap }}$, and $v=v_{0}$ until $v_{\text {sust }}$ is reached with increasing velocity (with decreasing time) resulting in $t_{2}$ and $s\left(t_{2}\right)$,

- vary $v_{\text {sust }}$ until $v_{\text {sust }}=\left[s\left(t_{2}\right)-s\left(t_{2}\right)\right] /\left[t_{2}-t_{2}\right]$, and

- vary $v_{0}$ in order to minimize energy at wheel.

The driving strategy for the Rockingham racing track obtained with this approach is illustrated in Figure 11. The sensitivity of the optimum to varying initial speeds is shown in Figure 12, whereas Figure 13 shows the dependency of the sustain speed for varying initial speeds. The sustain speed strongly depends on the initial speed, whereas the energy at the wheel varies only by approximately $1 \%$ in the range investigated. Thus it is important to choose the correct combination of initial and sustain speeds in order to meet the average-speed constraint. However, the proposed control strategy is robust with respect to variations in these parameters, i.e., the energy demand will not substantially increase even if the chosen initial and sustain speeds are suboptimal.
So far the behavior of the subsystem providing the propulsion energy has not been included in the optimization. All results obtained so far remain valid if this subsystem has a constant efficiency when transforming chemical energy stored in the hydrogen to mechanical energy that is absorbed by the traction wheel.

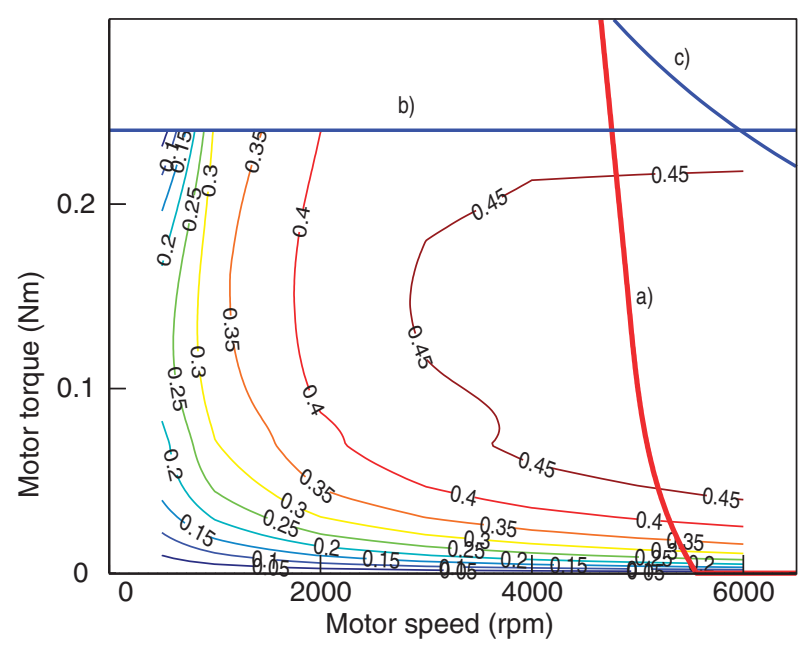

Figure 14

Tank-to-wheel efficiency map of the PAC Car II propulsion system. 


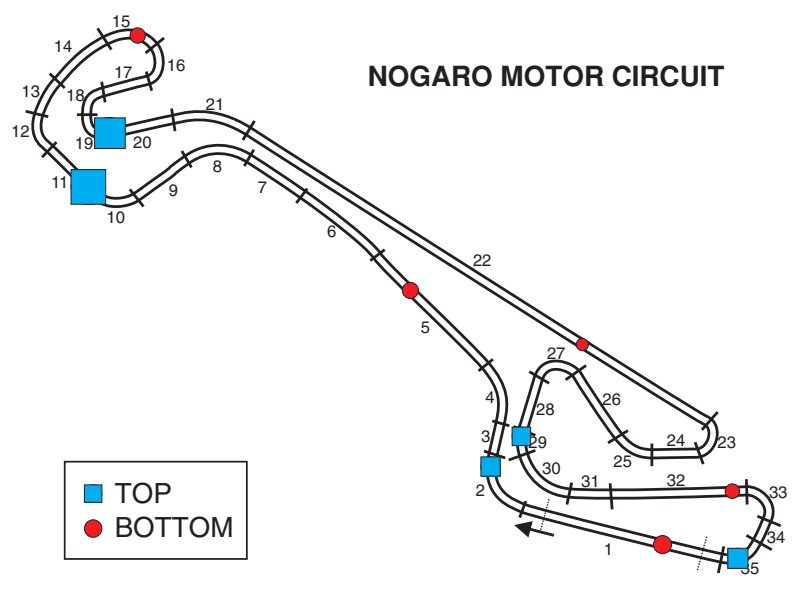

Figure 15

Layout and elevation information of the racing track in Nogaro (F).
Velocity $(m / s)$, traction force on wheel $(N)$, ext. forces $(N)$, elevation $(\mathrm{m})$

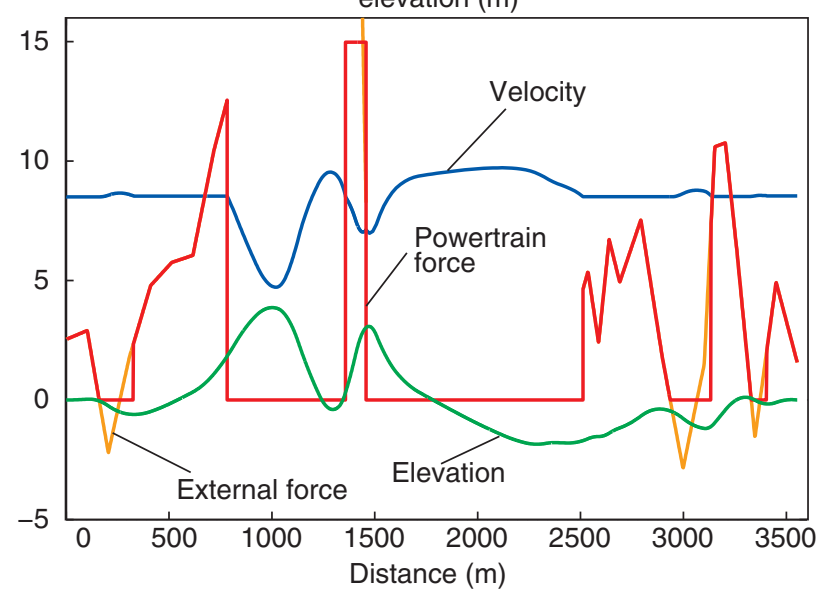

Figure 16

Driving profiles for the racing track in Nogaro $(F)$.
Fortunately, this condition is (approximately) satisfied for the fuel cell propulsion system of PAC Car II, as the efficiency map of Figure 14 shows. The gear ratio between motors and traction wheel must be chosen such that the operation limits are not violated and such that the propulsion system operates most of the time in the best-efficiency region (approximately at an efficiency of 0.45 in this example). The limits of operation are defined by the motor characteristics (current and power limits) and the polarization curve of the fuel cell. These limits are shown in Figure 14 as well.

Since PAC Car II is built for best aerodynamic performance, at narrow curves the vehicle may roll over if the speed is too high. Therefore, for a racing track with curves with small radii, the speed in the turns has to be limited as well. The track of Nogaro (F), whose main characteristics are shown in Figure 15, belongs to this class. If this restriction is taken into account, the space of possible solutions to the OCP is significantly reduced.

In Figure 15 positive elevations are indicated with a square, negative ones with a circle. The two critical curves are in the segments 16 and 23. In these curves the speed must not be higher than $10 \mathrm{~m} / \mathrm{s}$. Thus, the following strategy is chosen:

- drive with constant speed until the vehicle reaches that point from which, by shutting off the motor, the vehicle coasts to the appropriate speed at the boundary between segments 10 and 11 ,
- continue coasting until the sustain speed is again reached in the next ascent,

- since in this phase the grade is so steep that the speed cannot be held constant, use a short boost (maximum torque) phase here,

- after that, a coasting phase follows, such that the vehicle does not violate the top speed limitation at the transition point from segment 22 to 23 ,

- drive with constant speed to the end of the lap.

The elevation, the speed of the vehicle, the traction force, and the external force to the wheel are all illustrated in Figure 16. If the torque that the motors are supposed to produce is very low, a bad efficiency of the power train results (see Fig. 14). In such situations, the motors are shut off until the vehicle speed drops to $2 \%$ below the demanded value. Then, a short high-torque phase is commanded until the demanded speed is again reached. This duty-cycle mode is continued as long as necessary.

\section{CONCLUSION}

Mathematical models and model-based optimization can be useful to compute optimal feed forward control algorithms. Even though solutions are found often only for simplified problem settings, these results are excellent benchmarks for heuristic solutions that are developed to work in general settings. 


\section{REFERENCES}

1 Bryson E. and Ho Y.C. (1975) Applied Optimal Control. Taylor \& Francis, New York.

2 Wei X., Guzzella L., Utkin V., Rizzoni G. (2006) ModelBased Fuel-Optimal Control of Hybrid Electric Vehicle Using Variable Structure Control Systems. J. Dyn. Syst. - T. ASME, 129, 13-19.

3 Guzzella L., Amstutz A., Grob F. (1998) Optimal Operation Strategies for Hybrid Power trains, in Proc. 2nd IFAC
Workshop on Advances in Automotive Control, Mohican State Park, Ohio.

4 Guzzella L., Sciarretta A. (2005) Vehicle Propulsion Systems - Introduction to Modeling and Optimization. Springer Verlag, Berlin.

5 Guzzella L., Onder, Ch. (2006) Past, Present and Future of Automotive Control, in Control of Uncertain Systems: Modelling, Approximation, and Design, B. Francis and M. Smith (Eds.), Springer Verlag, Berlin.

Final manuscript received in December 2006 